\title{
Bedeutung nosokomialer Infektionen bei der Resektion trachealer Stenosen nach Tracheotomie ${ }^{1}$
}

\author{
Influence of Nosocomial Infections on Resection of Tracheal Stenosis \\ After Tracheotomy
}

Autoren

Institut
A. Wolter, C. Ludwig, F. Beckers, E. Stoelben

Lungenklinik Merheim, Zentrum für Thoraxchirurgie, Pneumologie/Onkologie und Schlaf- und Beatmungsmedizin, Kliniken der Stadt Köln gGmbH (Leiter: Prof. Dr. med. Erich Stoelben) $\begin{array}{lll}\text { eingereicht } & \text { 12.9. } 2011\end{array}$ akzeptiert nach Revision 20. 9. 2011

\section{Bibliografie}

Dol http://dx.doi.org/ 10.1055/s-0031-1291431 Online-Publikation: 10.11.2011 Pneumologie 2012; 66: 7-11 (c) Georg Thieme Verlag KG Stuttgart · New York ISSN 0934-8387

Korrespondenzadresse Dr. med. Andreas Wolter Lungenklinik Merheim Zentrum für Thoraxchirurgie, Pneumologie/Onkologie und Schlaf- und Beatmungsmedizin Kliniken der Stadt Köln gGmbH Ostmerheimer Str. 200 51109 Köln

a_wolter@gmx.de

\section{Zusammenfassung \\ $\nabla$}

Hintergrund: Komplikationen am Tracheostoma sind häufig mit nosokomialen Infektionen kombiniert. Frage: Wie beeinflusst die Besiedelung mit multiresistenten Keimen das Operationsergebnis bei trachealen Resektionen?

Patienten und Methode: Zwischen 2005 und 2009 erfolgte bei 30 Patienten eine primäre End-zuEndresektion der Trachea nach Tracheotomie. Die demografischen Basisdaten, Diagnosezeitpunkt der Trachealstenose nach Dekanülierung, Typ der vorausgegangenen Tracheotomie wurden dokumentiert. Präoperativ erfolgte eine flexible Bronchoskopie mit Bronchialspülung. Bei allen Fällen erfolgte postoperativ eine inhalative Antibiotikatherapie.

Ergebnisse: In 16 Fällen lag ein Zustand nach plastischer Tracheostomie (PT) vor, in 14 Fällen nach perkutaner dilatativer Tracheotomie (PDT). In der präoperativ erfolgten Bronchialspülung ließ sich bei $64 \%$ eine bakterielle Kontamination nachweisen. Der Hauptkeim war mit 23\% ein multiresistenter Pseudomonas aeruginosa (MR). Als Langzeitkomplikationen traten insgesamt drei Fälle auf, von denen alle bakteriell kontaminiert waren. Schlussfolgerung: Eine bakterielle Besiedelung nach Langzeitintubation tritt häufig auf und stellt einen negativen Prädiktor für das Outcome von primären End-zu-End-Resektionen der Trachea dar. Eine prophylaktische postoperative Antibiotikatherapie kann die Kurz- und Langzeitergebnisse verbessern.

\section{Einleitung}

\section{$\nabla$}

Der durchschnittliche innere Durchmesser der Trachea eines Erwachsenen beträgt $2,5 \mathrm{~cm}$. Eine klinisch signifikante Stenose tritt bei einer Reduktion des Durchmessers um mehr als 50\% auf [1]. Die Trachealstenose als klinisch bedeutsamste Langzeitkomplikation nach Tracheotomie kann

\section{Abstract \\ $\nabla$}

Background: Complications located at the tracheostoma often occur in combination with nosocomial infections. We posed the question: how is the surgical result in tracheal resections influenced by bacterial contamination with multiresistant germs?

Patients and Methods: Between 2005 and 2009 we performed a primary end-to-end-resection of the trachea after tracheotomy in 30 patients. The demographic basic data, the diagnostic data on tracheal stenosis after decanullation and type of tracheotomy were documented. Preoperatively all patients underwent a flexible bronchoscopy with bronchial lavage. All patients received an antibiotic inhalation therapy postoperatively.

Results: 16 patients presented a status post-permanent tracheotomy (PT), in 14 cases after percutaneous dilatative tracheotomy (PDT). In $64 \%$ of all cases the preoperative bronchial lavage was positive for bacterial contamination. The major pathogen was with $23 \%$ a multiresistant Pseudomonas aeruginosa (MR). In three cases long-termcomplications occurred, all of which were bacterially contaminated.

Conclusion: After long-term intubation a bacterial contamination is very common and presents a negative predictor for the outcome of primary tracheal end-to-end resections. A prophylactic postoperative antibiotic therapy can improve the short- and long-term results.

zu lebensgefährlichen Komplikationen führen. Die Inzidenz einer Stenose nach Tracheotomie wird in der Literatur zwischen $0-21 \%$ angegeben.

\footnotetext{
1 4/2011 als Vortrag gehalten auf dem 52.Kongress der Deutschen Gesellschaft für Pneumologie und Beatmungsmedizin e.V. (DGP) in Dresden sowie 5/2011 auf dem 128. Kongress der Deutschen Gesellschaft für Chirurgie (DGCH) im ICM München.
} 
Klinisch relevant und interventionsbedürftig sind hierbei jedoch nur 1 -2\% [2,3]. Nichtinvasive Maßnahmen, wie intermittierende Dilatationsmaßnahmen, Lasertherapie, temporäre oder permanente Stentimplantation, gelten eher als in ihrer Effektivität limitierte Alternativoptionen. Seit den 70er-Jahren etablierte sich, durch die Arbeiten von Grillo und Pearson vorangetrieben, die komplette Resektion des stenosetragenden Bereichs mit primärer End-zu-End-Resektion als Goldstandard in der chirurgischen Behandlung wegen ihrer hohen Erfolgsrate und geringen Morbidität [4].

Studien über Trachealresektionen umfassen in der Regel kleine Fallzahlen. Ausführliche Berichte zum Management sowie Multivarianzanalysen zu Prognosefaktoren in Bezug auf Anastomosenkomplikationen fehlen meist.

Die beste nichtinvasive diagnostische Maßnahme zur direkten visuellen Beurteilung des Stenosegrads der Trachealstenose ist die flexible fiberoptische Bronchoskopie.

Während der Beurteilung der Trachealstenose sind weitere Maßnahmen wie Biopsien zum Ausschluss eines Tumors oder eine Bronchiallavage zur Keimasservation problemlos möglich.

Bislang existieren nur sehr wenige Berichte zur genaueren Untersuchung der nosokomialen Keimbelastung nach Tracheotomie im Rahmen einer Langzeitbeatmung.

In unserer Studie untersuchen wir die Zusammensetzung des Keimspektrums generell sowie den Anteil multiresistenter Keime und eruieren, ob ein positiver Nachweis als negativer Prädiktor für das Outcome einer primären End-zu-End-Resektion der Trachea anzusehen ist. Die Studie ist retrospektiv und bezieht sich auf Fallzahlen in einem Thoraxchirurgiezentrum in Deutschland.

\section{Patienten und Methode \\ $\nabla$}

Es handelt sich um eine retrospektive Analyse von 30 Patienten, bei denen eine primäre End-zu-End-Resektion der Trachea wegen Trachealstenose nach Tracheotomie im Rahmen einer Langzeitintubation in der Lungenklinik Köln-Merheim, Abteilung für Thoraxchirurgie im Zeitraum von Februar 2005 bis Oktober 2009 durchgeführt wurde. In allen Fällen erfolgte fünf Tage präoperativ eine flexible fiberoptische Bronchoskopie zur visuellen Beurteilung des Stenosegrads, der Höhe der Stenose bzw. Lokalisation der Stenose in Bezug auf die Stimmbänder und den Ringknorpel, Länge der Stenose sowie sieben Tage postoperativ zur Beurteilung der trachealen Anastomosenheilung. In Einzelfällen erfolgte eine Computertomografie des Thorax ( $\bullet$ Abb.1). Des Weiteren erfolgte während dieser Untersuchung eine Bronchiallavage (BL) zur Keimasservation. Beim operativen Vorgehen zur Sanierung der Trachealstenose handelt es sich um eine inkomplette Resektion der Trachea, die nur die Vorderwand beinhaltet mit spannungsfreier End-zu-End-Resektion der Trachea. Das Resektionsausmaß erstreckt sich bis zur ersten gesunden Knorpelspange $($ Abb. 2 und $\bullet$ Abb.3). Es schließt sich eine Schleimhautdarstellung mit ausschließlicher Resektion der Mucosa der Hinterwand der Trachea an um ein Verletzungsrisiko des Nervus recurrens zu minimieren. Die End-zu-End-Anastomosennaht erfolgt fortlaufend mit PDS 4-0 ( $\bullet$ Abb.4). Bei allen Fällen erfolgte postoperativ eine Inhalation mit Tobramycin ${ }^{\circledR}$ für sieben Tage 80 mg morgens und abends. Die demografischen Basisdaten, der Typ der vorausgegangenen Tracheotomie, der Diagnosezeitpunkt der Trachealstenose nach Dekanülierung in Monaten, OP-Dauer, die Anzahl der tangentialen Ringknorpelresektionen sowie die Anzahl der Reinterventionen wurden dokumentiert.

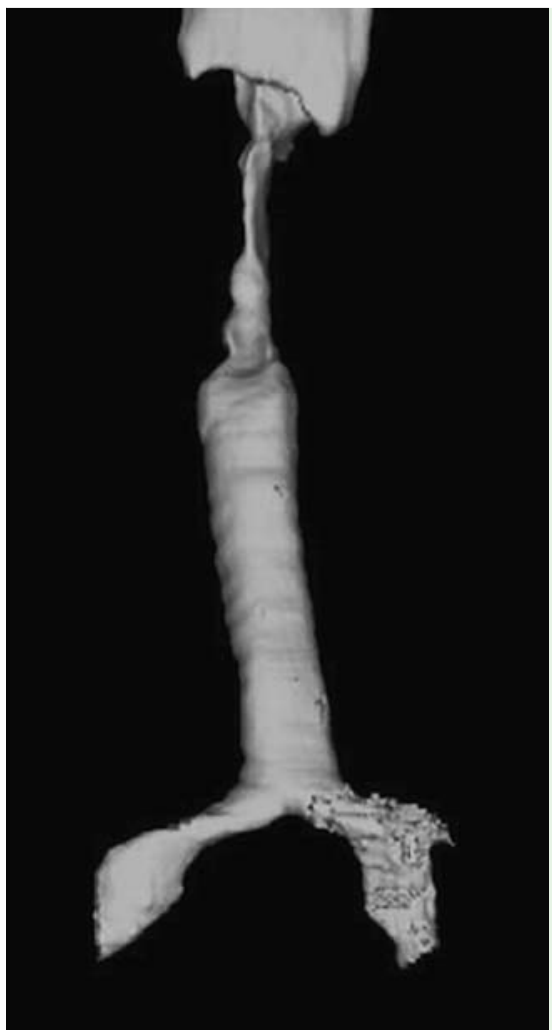

Abb. 1 Dreidimensionale CT-Rekonstruktion der Trachealstenose präoperativ.

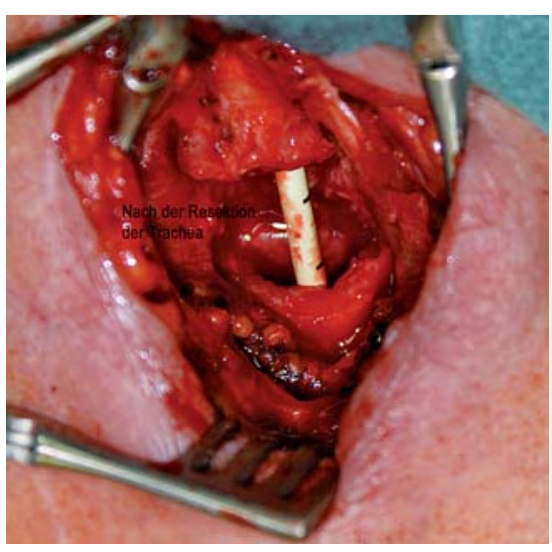

Abb.2 Intraoperativer Befund nach Resektion des stenosierten Tracheaanteils mit Jet-Ventilationskatheter in situ.

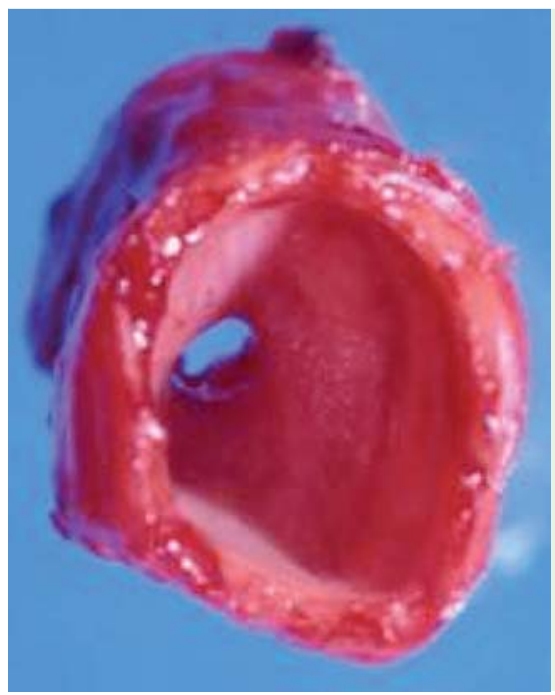

Abb.3 Resektionspräparat des stenosierten Tracheaanteils. 


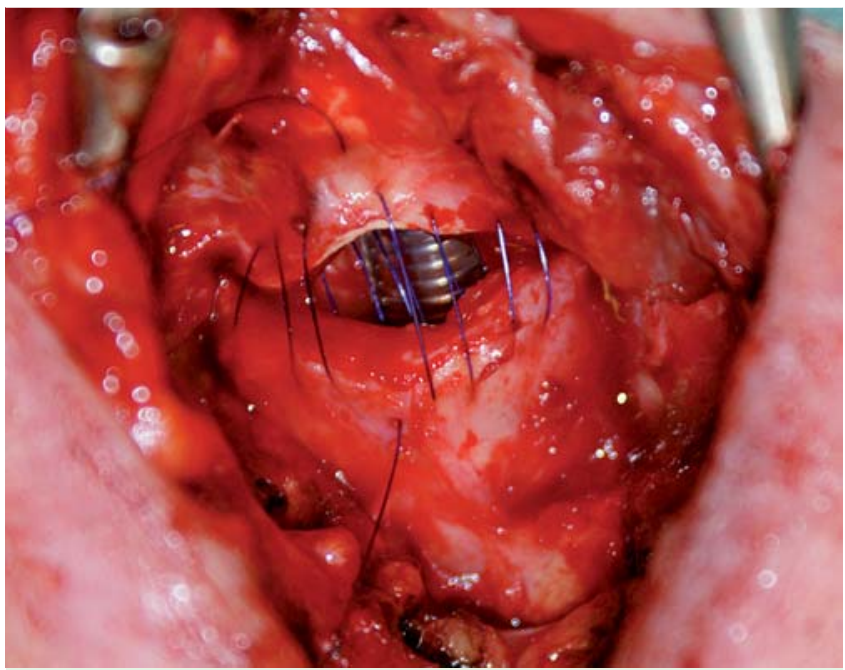

Abb.4 Fortlaufende End-zu-End-Anastomosennaht mit PDS 4-0.

Tab. 1 Patientenkollektiv, demografische Daten.

\begin{tabular}{|l|l|}
\hline Patienten und klinische Daten & \\
\hline $\mathbf{n}$ & $\mathbf{3 0}$ \\
\hline Alter (Jahre) & $62(38-82)$ \\
\hline Geschlecht & $18: 13$ (m:w) \\
\hline Z.n. Plastischer Tracheotomie (PT) & $53 \%$ \\
\hline Z.n. Dilatativer Punktionstracheotomie (PDT) & $47 \%$ \\
\hline CRP präop (mg/l) & $14(3-119)$ \\
\hline Stenosezeitpunkt nach Dekanülierung (Monate) & $45(1-312)$ \\
\hline Mittlerer Stenosegrad präoperativ & $60 \%$ \\
\hline Tangentiale Resektion des Ringknorpels & $27 \%$ \\
\hline Trachearesektion (End-zu-End) OP-Dauer (min) & $67(28-112)$ \\
\hline Wundinfektionen & $0 \%$ \\
\hline Langzeitkomplikationen & $10 \%$ \\
\hline
\end{tabular}

\section{Ergebnisse}

In 16 Fällen zeigte sich eine Trachealstenose nach vorausgegangener plastischer Tracheostomie (PT), in 14 Fällen nach perkutaner dilatativer Tracheotomie (PDT). In der präoperativ erfolgten Bronchoskopie ließ sich in der Gesamtpatientenzahl bei $64 \%$ eine bakterielle Kontamination nachweisen. Das Alter lag im Durchschnitt bei 62 Jahren. Die Geschlechtsverteilung war mit 18 Männern gegenüber 13 Frauen etwa gleichwertig. Der Diagnosezeitpunkt der interventionsbedürftigen Trachealstenose nach Dekanülierung lag im Mittel bei 45 Monaten. Eine tangentiale Resektion des Ringknorpels war in acht Fällen erforderlich ( Tab.1). Die Anastomose heilte in allen Fällen primär. Der in der präoperativ durchgeführten Bronchoskopie nachgewiesene Hauptkeim war mit 23\% ein multiresistenter (MR) Pseudomonas aeruginosa. Staphylococcus aureus war mit 16\% nachweisbar (davon $7 \% \mathrm{MRSA}$ ), Enterobacter cloacae (ESBL) zeigte sich in $10 \%$, koagulase-negative Staphylokokken mit 7\%, mit jeweils $3 \%$ andere Keime wie Streptococcus maltophilia, Hämophilus influenzae, vergrünende Streptokokken und Serratia marcescens (MR)

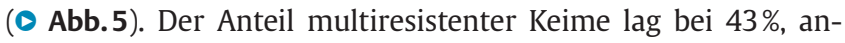
teilsmäßig vertreten waren mit 23\% Pseudomonas aeruginosa (MR), 10\% Enterobacter cloacae (ESBL), 7\% Staphylococcus aureus (MRSA) sowie mit 3\% Serratia marcescens (MR) ( $\bullet$ Abb.6).

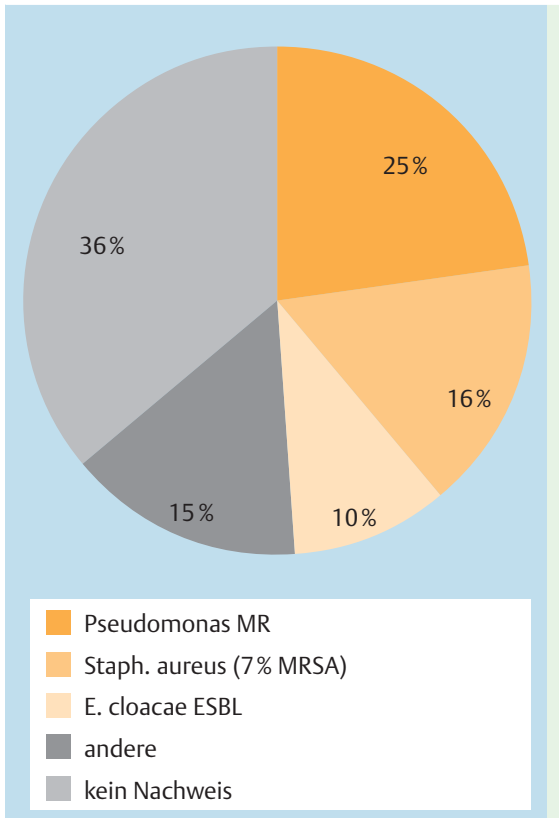

Abb. 5 Keimnachweis durch Bronchiallavage (BL) präoperativ.

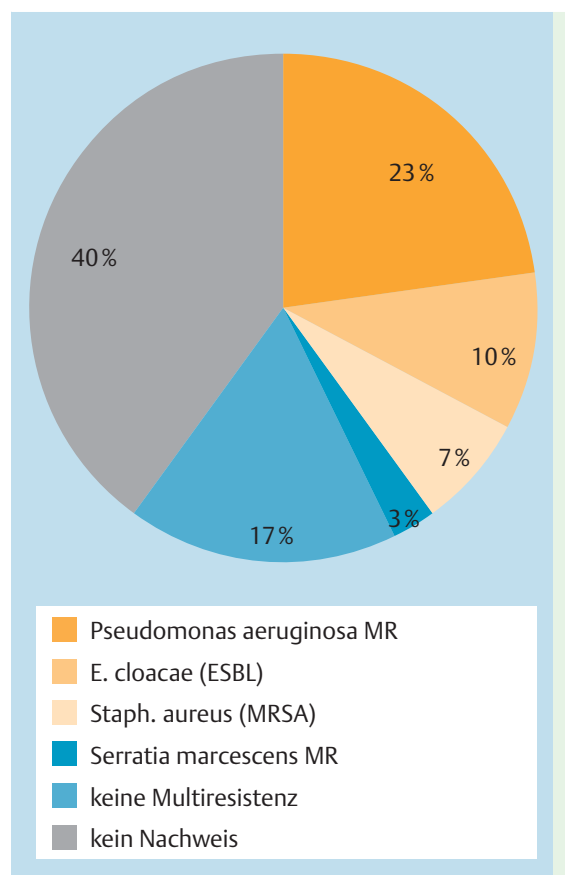

Abb.6 Anteil multiresistenter Keime (MR) in BL.

Als Langzeitkomplikationen traten zwei Restenosen auf. Im ersten Fall erfolgte eine Revisionsoperation mit Re-Anastomosierung. Bei dieser Patientin lag eine schwere COPD vor. Die Restenose trat mehrere Monate postoperativ auf. Mikrobiologisch bestand eine Resistenz von Pseudomonas aeruginosa gegenüber Tobramycin ${ }^{\circledR}$. Im zweiten Fall war eine Laserabtragung als Therapie ausreichend. Im dritten Fall zeigte sich eine Osteomyelitis des Sternums durch MRSA mit Notwendigkeit der Sternotomie. Bei diesen Fällen erfolgte eine perkutane dilatative Tracheotomie (PDT) im Vorfeld. Bei allen drei Fällen konnte in der präoperativen Bronchoskopie ein positiver Keimnachweis erbracht werden. 


\section{Diskussion}

7

In dieser Studie sollte die Bedeutung einer nosokomialen Keimkontamination als Einflussfaktor bei der Resektion trachealer Stenosen nach Tracheotomie untersucht werden. Andere Studien zeigten bereits, dass ein Vorhandensein von Diabetes mellitus, eine Reoperation, ein vergrößertes Resektionsausmaß und laryngotracheale Resektionen häufig mit Anastomosenkomplikationen assoziiert sind [5]. Diabetes spielt als negativer Prädiktor eine wichtige Rolle aufgrund der eingeschränkten Mikrozirkulation und damit resultierender verzögerter Wundheilung. Eine Reoperation stellt einen Risikofaktor dar, da durch die bereits erfolgte Resektion von Trachealgewebe erhöhte Spannungskräfte an der Anastomosennaht wirken. Grillo et al. beschrieben eine Zunahme an Anastomoseninsuffizienzen wegen exponentiell steigenden Spannungskräften bei zunehmender Resektionslänge $>4 \mathrm{~cm}$ des stenosetragenden Bereichs der Trachea [6]. Hierbei können keine Guidelines zur maximal möglichen Resektionslänge gegeben werden, jedoch Empfehlungen für eine schrittweise Rekonstruktion der Trachea bei Resektionslängen $>4 \mathrm{~cm}$ [7].

Patienten mit einer Trachealstenose nach Tracheotomie zeigen oft eine peritracheale Entzündungsreaktion distal des maximalen Stenosebereichs, welche die Mobilität für eine Reapproximierung limitiert. Eine laryngotracheale Anastomosierung bezieht sich auf zwei Luftwegsareale und zeigt im Vergleich zu einer tracheotrachealen Anastomosierung eine erhöhte Rate an Anastomoseninsuffizienzen [6].

Raghuraman et al. beschrieben 2005 eine schneller entstehende Tracheastenose nach vorausgegangener perkutaner dilatativer Tracheotomie (PDT) gegenüber plastischer Tracheotomie (PT) [8]. Des Weiteren war bei den PDT-Fällen die Rekonstruktion der Trachea schwieriger, bedingt durch die häufige Lokalisierung im subglottischen Bereich. Die Stenose lag bei den PDT-Fällen im Durchschnitt höher in Nähe der Stimmlippen und zeigte oft eine Knorpelfrakturierung mit zusätzlicher Lumenbeeinträchtigung. Die Inzidenz von Knorpelfrakturen nach PDT wird in der Literatur mit $87 \%$ angegeben, bei PT beträgt sie ca. 2\% [9]. Muttini et al. hingegen beschrieben bei Durchführung einer dilatativen Tracheotomie unter bronchoskopischer Kontrolle in einem versierten Zentrum eine geringere Interventionsdauer für die dilatative Tracheotomie und eine geringere Rate für frühpostoperative Infektionen sowie ein besseres kosmetisches Ergebnis [10].

Eine frühzeitige Diagnosestellung einer Anastomoseninsuffizienz durch eine postoperative Bronchoskopie vor Entlassung kann weitere schwerwiegende Komplikationen verhindern. Die mit einer trachealen Anastomoseninsuffizienz assoziierte Mortalitätsrate wird in der Literatur mit ca. 7\% angegeben [5]. Seit 1988 starb kein Patient an einer Anastomoseninsuffizienz. Dies reflektiert die frühzeitige Diagnose und das verbesserte Handlungsregime dieser Komplikation. Diese Studie besitzt Schwächen zum einen dadurch, dass sie retrospektiv ist und sich auf Patientenfälle in einer einzigen Institution bezieht. Spezifisch bedeutsame Selektionsfaktoren als Prädiktoren im Rahmen einer Trachearesektion nach Tracheotomie sind schwer zu quantifizieren und identifizieren.

Eine nosokomiale Keimbesiedelung mit Differenzierung des Keimspektrums als Prädiktor wurde bislang nur selten in der Literatur beschrieben. Brook differenzierte 1987 die bakterielle Kontamination nach Tracheotomie bei Kindern mit Beatmungsdauern zwischen 3 bis 12 Monaten. Hierbei war eine Mischbesiedelung aus aeroben und anaeroben Keimen nachweisbar gewesen, von denen $72 \%$ der Bakterien das Enzym Beta-Laktamase produzierten [11]. Von Arola wurde bei 794 tracheotomierten erwachsenen Patienten mit $92 \%$ eine bakterielle Besiedelung nachgewiesen [12]. In unserer Studie konnte durch eine präoperative fiberoptische Bronchoskopie das Keimspektrum in unserem Patientenkollektiv eruiert werden. Dabei stellte sich mit $64 \%$ ein relativ hoher Anteil an bakterieller Kontamination in der Gesamtzahl der Patienten heraus.

Der Hauptkeim hierbei war mit 23\% ein multiresistenter Pseudomonas aeruginosa. Die Bronchoskopie wird vor dem Eingriff durchgeführt zur Beurteilung der Stenose vor der Resektion. Die erhobene Mikrobiologie zeigt, mit welchen Keimen der Patient präoperativ besiedelt ist ohne ein Vorhandensein einer akuten Infektion. Die frühe postoperative Phase ist für die Anastomosenheilung entscheidend [13]. Eine postoperative prophylaktische Vernebelungsinhalation mit einem Antibiotikum kann eine Tendenz zur verbesserten Heilung zeigen [14]. In unserer Studie erfolgte eine postoperative Inhalation mit Tobramycin ${ }^{\circledR}$. Gegebenenfalls wurde nach erfolgtem Antibiogramm die Antibiose angepasst. Trotz der hohen Keimnachweisrate war eine Reintervention nur in drei Fällen erforderlich. Bei diesen Fällen zeigte sich jeweils ein positiver Keimnachweis präoperativ. Somit kann eine nosokomiale Kontamination als negativer Prädiktor betrachtet werden. Die präoperative Bronchoskopie dient in erster Linie zur Planung des operativen Eingriffs. Hierbei ist nicht nur die genaue Beurteilung des Stenosegrads und der Stenoseverhältnisse möglich, sondern sie eröffnet auch die Möglichkeit der Keimbestimmung, um bei positivem Nachweis einer möglichen Anastomoseninsuffizienz prophylaktisch antibiotisch entgegenwirken zu können.

\section{Schlussfolgerung \\ $\nabla$}

Generell sollte jeder Trachearesektion eine präoperative diagnostische fiberoptische Bronchoskopie mit mikrobiologischer Keimbestimmung vorausgehen. Eine nosokomiale Kontamination nach Langzeitintubation im Rahmen einer Tracheotomie kann als negativer Prädiktor für das Outcome von primären End-zuEnd-Resektionen der Trachea angesehen werden. Eine prophylaktische postoperative Antibiotikatherapie kann die Kurz- und Langzeitergebnisse verbessern.

\section{Interessenkonflikt}

$\nabla$

Die Autoren geben an, dass kein Interessenkonflikt besteht.

\section{Literatur}

1 Streitz JM, Shapshay SM. Airway injury after tracheotomy and endotracheal intubation. Surg Clin North Am 1991; 71: 1211 - 1230

2 Norwood S, Vallina VL, Short K et al. Incidence of Tracheal Stenosis and Other Late Complications after Percutaneous Tracheostomy. Ann Surg 2000; 232: 233-241

3 Tsitouridis I, Michaelides M, Dimarelos Vet al. Endotracheal and tracheostomy tube-related complications: imaging with three-dimensional spiral computed tomography. Hippokratia 2009; 13: 97-100

4 Grillo HC, Dignan EF, Miura T. Extensive resection and reconstruction of the mediastinal trachea without prosthesis or graft: an anatomical study in man. J Thorac Cardiovasc Surg 1964; 48: 741 - 749

5 Wright $C D$, Graham BB, Grillo HC et al. Anastomotic complications after tracheal resection: Prognostic factors and management. J Thorac Cardiovasc Surg 2004; 128: 731 -739

6 Grillo HC, Donahue DM, Mathisen DJ et al. Postintubation tracheal stenosis. Treatment and results. J Thorac Cardiovasc Surg 1995; 109: 486-493 
7 Biller HF, Lawson $W$, Weisberg $V$. Staged repair of extensive tracheal and laryngotrachealstenoses. Ann Otol Rhinol Laryngol 1986; 95: 586589

8 Raghuraman G, Rajan S, Marzouk JK et al. Is Tracheal Stenosis Caused by Percutaneous Tracheostomy Different From That by Surgical Tracheostomy? Chest 2005; 127: 879-885

9 Van Heurn LWE, Theunissen PHMH, Ramsay G et al. Pathological changes of the trachea after percutaneous dilatational tracheotomy. Chest 1996; 109: 1466 - 1469

10 Muttini S, Melloni G, Gemma M et al. Percutaneous or surgical trachetomy. Prospective, randomized comparison of the incidence of early and late complications. Minerva Anestesiol 1999; 65: 521 - 527
11 Brook I. Microbiological studies of tracheostomy site wounds. Eur J Respir Dis 1987; 71: 380-383

12 Arola MK. Tracheostomy and its complications. A retrospective study of 794 tracheostomized patients. Ann Chir Gynaecol 1981; 70: 96-106

13 Stoelben E, Harpering H, Haberstroh J et al. Heterotopic transplantation of cryopreserved tracheae in a rat model. Eur J Cardiothorac Surg 2003; 23: $15-20$

14 Ludwig C, Riedel R, Schnell J et al. Inhalation with Tobramycin ${ }^{\circledR}$ to improve healing of tracheobronchial reconstruction. Eur J Cardiothorac Surg 2009; 35: 797-800 